\title{
Religionszugehörigkeiten in Österreich auf Bundesländerebene: Schätzungen für Vorarlberg, 2001-2018
}

\author{
Anne Goujon, Claudia Reiter und Michaela Potančoková ${ }^{1}$ \\ (deutsche Übersetzung von Teilen der englischen Langfassung ${ }^{2}$ )
}

Die Studie wurde von okay.zusammen leben/Verein Aktion Mitarbeit finanziell unterstützt.

\section{Zusammenfassung der Ergebnisse}

Religionszugehörigkeit findet heutzutage viel Beachtung in den öffentlichen Debatten in Österreich, sowohl in den Medien als auch im politischen Diskurs. In dieser Studie untersuchen wir nicht die schwer zu quantifizierende Religiosität (also die individuelle Ausprägung von Religion beim einzelnen Menschen), sondern den Wandel der Religionszugehörigkeit der Bevölkerung im Land Vorarlberg. Dazu bedient sich diese Studie wissenschaftlicher Methoden unter Verwendung verfügbarer Daten und Proxydaten, um die Verteilung der Bevölkerung nach Religionszugehörigkeit im Jahr 2018 zu schätzen. Die letzte Volkszählung, bei der religionsbezogene Daten erhoben wurden, fand im Jahr 2001 statt. Seitdem stützen sich die Bevölkerungszahlen auf Registerdaten, ohne aktuelle Daten über die Religionszugehörigkeit der österreichischen Bevölkerung $\mathrm{zu}$ erheben. Daher muss die Religionszugehörigkeit anhand von Bevölkerungsprojektionen nach einer Methodik geschätzt werden, die von Goujon et al. (2017) ${ }^{3}$ in einem Projekt zur Rekonstruktion (und Zukunftsprojektion) der Bevölkerung Österreichs und Wiens im Jahr 2016 entwickelt wurde.

Die Schätzung zeigt, dass in Vorarlberg ähnliche Trends zu beobachten sind wie in Österreich insgesamt:

- Der Anteil der Katholikinnen und Katholiken nimmt stark ab, von $79 \%$ der Gesamtbevölkerung im Jahr 2001 auf 61 \% im Jahr 2018, was auf starke Säkularisierungstendenzen und auf einen Anstieg anderer Religionsbekenntnisse in der Bevölkerung zurückzuführen ist.

- $\quad$ Es gibt einen starken Anstieg der Bevölkerung ohne Religionszugehörigkeit, der sich zwischen 2001 und 2018 mehr als verdreifacht hat und dessen Anteil an der Gesamtbevölkerung von $6 \%$ auf $18 \%$ gestiegen ist.

- In geringerem Maße als in Wien hat sich der Anteil der Musliminnen und Muslime zwischen 2001 und 2018 von 8,5\% auf $13 \%$ (von 30.000 auf 51.000 Personen) deutlich erhöht. Dies ist das Ergebnis zweier wichtiger Entwicklungen - Fertilität und Zuwanderung. Während sich die Fertilität in Österreich geborener muslimischer Frauen nicht von der Fertilität anderer in Österreich geborener Frauen unterscheidet, haben

\footnotetext{
1 Wittgenstein Centre for Demography and Global Human Capital (IIASA, VID/OeAW, WU), Österreich.

2 Englische Langfassung „Religious Affiliations in Austria at the Provincial Level: Estimates for Vorarlberg, 2001-2018“ verfügbar unter: https://www.oeaw.ac.at/fileadmin/subsites/Institute/VID/PDF/Publications/Working Papers/WP2018 13.pdf.

${ }^{3}$ Die Studie kann unter folgender Adresse eingesehen werden: https://www.integrationsfonds.at/publikationen/forschungsberichte/forschungsbericht-demographieund-religion/ [18. 09. 2018].
} 
muslimische Frauen, die außerhalb Österreichs geboren wurden, im Durchschnitt ein Kind mehr. Zudem waren Musliminnen und Muslime unter den Zugewanderten im Zeitraum 2011 bis 2015 stärker vertreten als in früheren Perioden seit 2001.

In Bezug auf die Herkunft (das Geburtsland) der muslimischen Bevölkerung Vorarlbergs im Jahr 2018 zeigt sich, dass der Anteil der Musliminnen und Muslime, die in Österreich geboren wurden, zugenommen hat. Dabei handelt es sich überwiegend um die Nachkommen von Personen, die aus der Türkei zugewandert sind. In jüngster Zeit sind aufgrund der Fluchtmigration die Zahl und der Anteil der Musliminnen und Muslime, die in anderen Ländern geboren wurden als in den traditionellen Herkunftsländern der muslimischen Zuwanderung nach Vorarlberg (d. h. in der Türkei, im ehem. Jugoslawien und in anderen europäischen Ländern), gestiegen. Durch diese jüngste Zuwanderung hat sich die muslimische Bevölkerung diversifiziert. Der Anteil derjenigen, die aus nicht-traditionellen Zuzugsländern (hauptsächlich dem Nahen und Fernen Osten) stammen, ist zwischen 2001 und 2018 von $1 \%$ auf $12 \%$ der muslimischen Bevölkerung gestiegen. 


\section{Einleitung}

Religionszugehörigkeit findet heutzutage viel Beachtung in den öffentlichen Debatten in Österreich, sowohl in den Medien als auch im politischen Diskurs. Dabei sind zweierlei Schwerpunkte zu erkennen: zum einen die jüngsten Migrationsbewegungen, verbunden mit der Frage, wie sie die religiöse Landschaft in Bezug auf die Anzahl der Glaubensangehörigen verändern, insbesondere im Hinblick auf den relativen Anteil der Musliminnen und Muslime an der Bevölkerung. Der zweite Schwerpunkt der öffentlichen Debatten liegt auf der Religiosität der muslimischen Glaubensangehörigen, seien es Zugewanderte oder in Österreich Geborene, und den gesellschaftlich kritischen Dimensionen, die dieser Religiosität zugeschrieben werden. In dieser Studie untersuchen wir nicht die Religiosität, die sich schwer quantifizieren lässt, sondern den Wandel der Religionszugehörigkeit der Bevölkerung (erster Schwerpunkt) im Land Vorarlberg. Dazu bedient sich diese Studie wissenschaftlicher Methoden unter Verwendung verfügbarer Daten und Proxydaten, um die Verteilung der Bevölkerung nach Religionszugehörigkeit im Jahr 2018 zu schätzen. Die letzte Volkszählung, bei der religionsbezogene Daten erhoben wurden, fand im Jahr 2001 statt. Seitdem stützen sich die Bevölkerungszahlen auf Registerdaten, ohne aktuelle Daten über die Religionszugehörigkeit der österreichischen Bevölkerung zu erheben. Daher muss die Religionszugehörigkeit anhand von Bevölkerungsprojektionen nach einer Methodik geschätzt werden, die von Goujon et al. (2017) ${ }^{4}$ in einem Projekt zur Rekonstruktion (und Zukunftsprojektion) der Bevölkerung Österreichs und Wiens im Jahr 2016 entwickelt wurde.

Im Jahr 2001 war die Vorarlberger Bevölkerung weitgehend auf drei Gruppen verteilt: Katholikinnen und Katholiken (79,2 \%), Musliminnen und Muslime (8,5\%) und Personen ohne Bekenntnis (6,1\%). Vorarlberg war das österreichische Bundesland mit dem höchsten Anteil muslimischer Bevölkerung (meist türkischer Herkunft) 5 . Diese Studie zielt darauf ab, die Frage nach den Auswirkungen der jüngsten Migrationsbewegungen, insbesondere der Zuwanderung von Asylbewerbenden aus Syrien, dem Irak und aus Afghanistan von 2015 bis 2016 (Buber-Ennser et al. 2016, 2018) auf die religiöse Zusammensetzung der Vorarlberger Bevölkerung zu beantworten. Im ersten Abschnitt präsentieren wir die Daten und Methoden zur Rekonstruktion der Bevölkerung bis ins Jahr 2018 anhand von sechs Religionszugehörigkeiten: römisch-katholisch, protestantisch, orthodox, muslimisch, andere Bekenntnisse und ohne Bekenntnis. Die Ergebnisse der Rekonstruktion werden im zweiten Abschnitt dargestellt, in dem wir auch Vorarlberg mit Österreich und Wien (2018) vergleichen.

\footnotetext{
${ }^{4}$ Die Studie kann unter folgender Adresse eingesehen werden: https:/www.integrationsfonds.at/publikationen/forschungsberichte/forschungsbericht-demographieund-religion/ [18. 09. 2018].

${ }_{5}$ Nach proportionaler Umverteilung derjenigen Personen, die in der Volkszählung ihre Religionszugehörigkeit nicht angeführt haben, auf unsere sechs Religionszugehörigkeiten betrug der Anteil der muslimischen Bevölkerung in Wien 8,2 \%, in Oberösterreich, Salzburg und Tirol rund 4-5 \%, in Niederösterreich 3,2 \% und im Burgenland, in Kärnten und in der Steiermark weniger als 2 \%.
} 


\section{Daten und Methodik}

Die Rekonstruktion der Bevölkerung basiert auf den neuesten verfügbaren Daten zur Religionszugehörigkeit, die bei der Volkszählung 2001 erhoben wurden. Sie folgt der demografischen Methodik der multi-state population projections, die eine Erweiterung des Projektionsmodells nach der Kohorten-Komponenten-Methode darstellt, das in der Regel für Bevölkerungsprojektionen verwendet wird.

Die Projektion von 2001 bis 2018 erfolgt in drei Schritten von 5-Jahres-Zeiträumen (2001-2005, 2006-2010, 2011-2015) und einem letzten 2-Jahres-Zeitraum (2016-2017) und benötigt die folgenden Angaben:

- Bevölkerung nach Alter, Geschlecht und Religion im Jahr 2001

- Fertilität nach Alter und Religion von 2001 bis 2017

- Mortalität nach Alter, Geschlecht und Religion von 2001 bis 2017

- Migration (Zu- und Abwanderung) nach Alter, Geschlecht und Religion von 2001 bis 2017

- Religionswechsel (meist im Sinne der Säkularisierung) nach Alter und Geschlecht von 2001 bis 2017

Nicht alle benötigten Angaben stehen in der erforderlichen Detailliertheit ohne Weiteres zur Verfügung. Daher mussten einige Schätzverfahren angewandt werden, um alle für die Projektionen bis 2018 notwendigen Annahmen zu treffen.

Eine detaillierte Beschreibung der methodischen Herangehensweise finden Sie in der englischen Version dieses Berichts. ${ }^{6}$

\footnotetext{
${ }^{6}$ Verfügbar unter:

https://www.oeaw.ac.at/fileadmin/subsites/Institute/VID/PDF/Publications/Working Papers/WP2018 13.pdf
} 


\section{Schätzungen zur Religionszugehörigkeit der Vorarlberger Bevölkerung im Jahr 2018}

Das Ergebnis der Schätzung zeigt, dass sich die religiöse Zusammensetzung der Bevölkerung in Vorarlberg zwischen 2001 und 2018 deutlich verändert hat. Die auffälligste Veränderung ist der Rückgang der Katholikinnen und Katholiken, sowohl in absoluten Zahlen als auch anteilsmäßig: von 79 \% im Jahr 2001 auf 61 \% im Jahr 2018. Dies ist zwei wesentlichen Entwicklungen geschuldet: zum einen dem Anstieg der Bevölkerung ohne Bekenntnis, die sich zwischen 2001 und 2018 mehr als verdreifacht hat und deren Anteil an der Gesamtbevölkerung von $6 \%$ auf $18 \%$ gestiegen ist; zum andern den Auswirkungen der Zuwanderung, durch die sich die Anteile der protestantischen, orthodoxen und muslimischen Bevölkerung erhöht haben. Insbesondere der Anteil der Musliminnen und Muslime ist zwischen 2001 und 2018 von 8,5\% auf 13 \% gestiegen (von 30.000 auf 51.000 Personen).

Abbildung 10: Bevölkerung Vorarlbergs nach Religionszugehörigkeit in absoluten Zahlen, 20012018

\begin{tabular}{|c|c|c|c|c|c|c|}
\hline & 50,000 & $100,000 \quad 150,000$ & 200,000 & 250,000 & 300,000 & $350,000 \quad 400,000$ \\
\hline \multicolumn{7}{|l|}{2018} \\
\hline \multicolumn{7}{|l|}{2016} \\
\hline \multicolumn{7}{|l|}{2011} \\
\hline \multicolumn{7}{|l|}{2006} \\
\hline \multicolumn{7}{|l|}{2001} \\
\hline & 2001 & 2006 & 2011 & & 2016 & 2018 \\
\hline Römisch-katholisch & 277,402 & 269,693 & 256,543 & & 243,124 & 239,040 \\
\hline Protestantisch & 7,908 & 8,685 & 9,620 & & 10,755 & 11,376 \\
\hline Orthodox & 9,270 & 10,798 & 11,817 & & 14,228 & 15,618 \\
\hline Muslimisch & 29,812 & 36,113 & 39,939 & & 47,693 & 50,797 \\
\hline Andere Bekenntnisse & 4,486 & 4,917 & 5,197 & & 5,775 & 6,050 \\
\hline Ohne Bekenntnis & 21,250 & 32,424 & 46,184 & & 62,572 & 68,860 \\
\hline
\end{tabular}

Quelle: Berechnung der Autorinnen auf der Grundlage von Daten der Statistik Austria

Hinweis: Die Gesamtzahl der Katholikinnen und Katholiken in Abbildung 10 unterscheidet sich von den Mitgliederzahlen der Katholischen Kirche in Vorarlberg, da nicht alle Personen, die sich als "katholisch“ deklarieren (wie in der Volkszählung), notwendigerweise Mitglieder der katholischen Kirche sind. Die Differenz beträgt 2016 etwa 2 \%, wie zum Zeitpunkt der Volkszählung im Jahr 2001. 
Abbildung 11: Anteil der Vorarlberger Bevölkerung nach Religionszugehörigkeit, 2001-2018

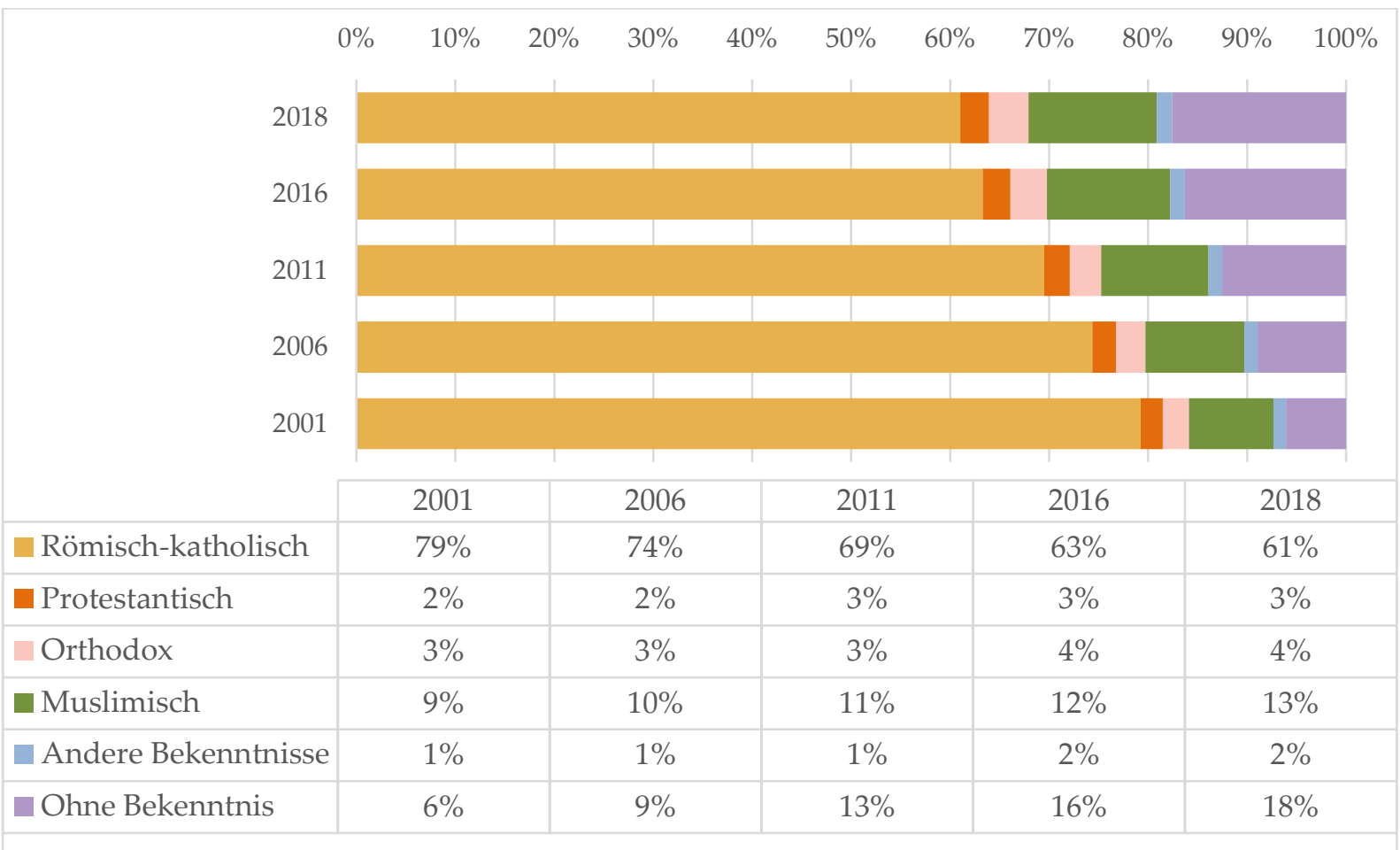

Quelle: Berechnung der Autorinnen auf der Grundlage von Daten der Statistik Austria

\subsection{Vergleich mit der Religionszugehörigkeit in Österreich und in Wien}

Im Jahr 2017 veröffentlichten Goujon et al. Schätzungen der Religionszugehörigkeiten der Bevölkerung Österreichs und Wiens im Jahr 2016 sowie eine Projektion bis zum Jahr 2046. Wir vergleichen in der folgenden Tabelle (Tabelle 4) diese Ergebnisse (interpoliert zwischen 2016 und 2021 - es gibt keinen signifikanten Unterschied zwischen den Szenarien im Jahr 2018) mit denen der vorliegenden Arbeit.

Tabelle 4: Religiöse Zusammensetzung der Bevölkerung in Österreich, Wien und Vorarlberg 2001 (Volkszählung) und 2018 (Rekonstruktion)

\begin{tabular}{|l|rr|rr|rr|}
\cline { 2 - 7 } \multicolumn{1}{c|}{} & \multicolumn{2}{c|}{ Österreich } & \multicolumn{2}{c|}{ Wien } & \multicolumn{2}{c|}{ Vorarlberg } \\
\hline Religionsbekenntnisse & 2001 & 2018 & 2001 & $2018^{*}$ & 2001 & 2018 \\
\hline Römisch-katholisch & $75 \%$ & $63 \%$ & $49 \%$ & $34 \%$ & $79 \%$ & $61 \%$ \\
\hline Protestantisch & $5 \%$ & $5 \%$ & $5 \%$ & $4 \%$ & $2 \%$ & $3 \%$ \\
\hline Orthodox & $2 \%$ & $5 \%$ & $6 \%$ & $10 \%$ & $3 \%$ & $4 \%$ \\
\hline Muslimisch & $4 \%$ & $8 \%$ & $8 \%$ & $15 \%$ & $9 \%$ & $13 \%$ \\
\hline Andere Bekenntnisse & $1 \%$ & $2 \%$ & $7 \%$ & $6 \%$ & $1 \%$ & $2 \%$ \\
\hline Ohne Bekenntnis & $12 \%$ & $18 \%$ & $26 \%$ & $30 \%$ & $6 \%$ & $18 \%$ \\
\hline
\end{tabular}

${ }^{*}$ Interpoliert zwischen 2016 und 2018.

Quelle: Goujon et al. 2017; Berechnung der Autorinnen auf der Grundlage von Daten der Statistik Austria 
Vorarlberg weist Ähnlichkeiten mit Österreich und Wien auf. Der Anteil der Katholikinnen und Katholiken im Jahr 2001, der in Vorarlberg nahe am Bundesdurchschnitt lag (79 \% vs. $75 \%$ ), sank in den 17 Jahren der Beobachtung sowohl in Vorarlberg als auch in ganz Österreich deutlich (61 \% vs. 63 \% im Jahr 2018). Der Anteil der muslimischen Bevölkerung lag in Vorarlberg etwa auf dem gleichen Niveau wie in Wien (9\% vs. $8 \%$ im Jahr 2001). Die Schätzungen scheinen zu zeigen, dass der Anstieg der muslimischen Bevölkerung in der Wiener Bevölkerung etwas stärker ausfiel, da 2018 etwa 15 \% der Wiener Bevölkerung eine muslimische Religionszugehörigkeit haben, verglichen mit $13 \%$ in Vorarlberg. Der Anteil der Bevölkerung ohne Bekenntnis war 2001 in Vorarlberg (6\%) deutlich niedriger als in Österreich $(12 \%)$ und Wien $(26 \%)$, hat sich aber seitdem verdreifacht und beträgt 2018 für diese Bevölkerungsgruppe $18 \%$, womit er das gleichen Niveau wie in ganz Österreich erreichte.

\section{Geschätzte Verteilung der Herkunftsländer der muslimischen Bevölkerung in Vorarlberg im Jahr 2018}

\subsection{Methodik}

Um die Verteilung der Herkunftsländer der muslimischen Bevölkerung in Vorarlberg im Jahr 2018 zu schätzen, werden ihre Geburtsländer (GL) in fünf Kategorien unterteilt:

- in Österreich geborene Musliminnen und Muslime (hauptsächlich Nachkommen von Zugewanderten; zweite und dritte Generation)

- in der Türkei geborene Musliminnen und Muslime

- Musliminnen und Muslime, die in Ländern des ehemaligen Jugoslawiens geboren wurden (Bosnien und Herzegowina, Kroatien, Kosovo, Mazedonien, Montenegro, Serbien, Slowenien)

- Musliminnen und Muslime, die in Ländern der Europäischen Union geboren wurden (außer Österreich, Kroatien und Slowenien, da diese in anderen Kategorien enthalten sind)

- in anderen Ländern geborene Musliminnen und Muslime

\subsection{Die muslimische Bevölkerung in Vorarlberg im Jahr 2001}

Laut der Volkszählung von 2001 lebten 29.334 Musliminnen und Muslime in Vorarlberg. Abbildung 12 zeigt die Verteilung der muslimischen Bevölkerung nach Alter, Geschlecht und Geburtsland-Kategorie im Mai 2001. Etwa die Hälfte der damals in Vorarlberg lebenden muslimischen Glaubensangehörigen wurde in der Türkei geboren; weitere 38 \% wurden in Österreich geboren. Jene Musliminnen und Muslime, die in Österreich geboren wurden, waren zumeist zwischen 0 und 24 Jahre alt, was darauf hindeutet, dass es bereits eine beträchtliche Zahl von Zugewanderten der zweiten Generation gab. Weitere 11 \% stammten ursprünglich aus den Ländern des ehemaligen Jugoslawiens, wobei die Mehrheit von ihnen in Bosnien und Herzegowina geboren wurde. Der Rest (ca. 2 \%) wurde in anderen Ländern geboren.

Im Vergleich zur Alters- und Geschlechterverteilung der Gesamtbevölkerung Vorarlbergs im Jahr 2001 war die muslimische Bevölkerung deutlich jünger und hatte einen höheren 
Männeranteil: Während das mediane Lebensalter der gesamten Vorarlberger Bevölkerung im Jahr 2001 in der Altersgruppe 35-39 Jahre lag, war es bei der muslimischen Bevölkerung in der Altersgruppe 25-29 Jahre. Darüber hinaus waren 55 \% der 2001 in Vorarlberg lebenden muslimischen Glaubensangehörigen männlich, gegenüber 49 \% der Gesamtbevölkerung.

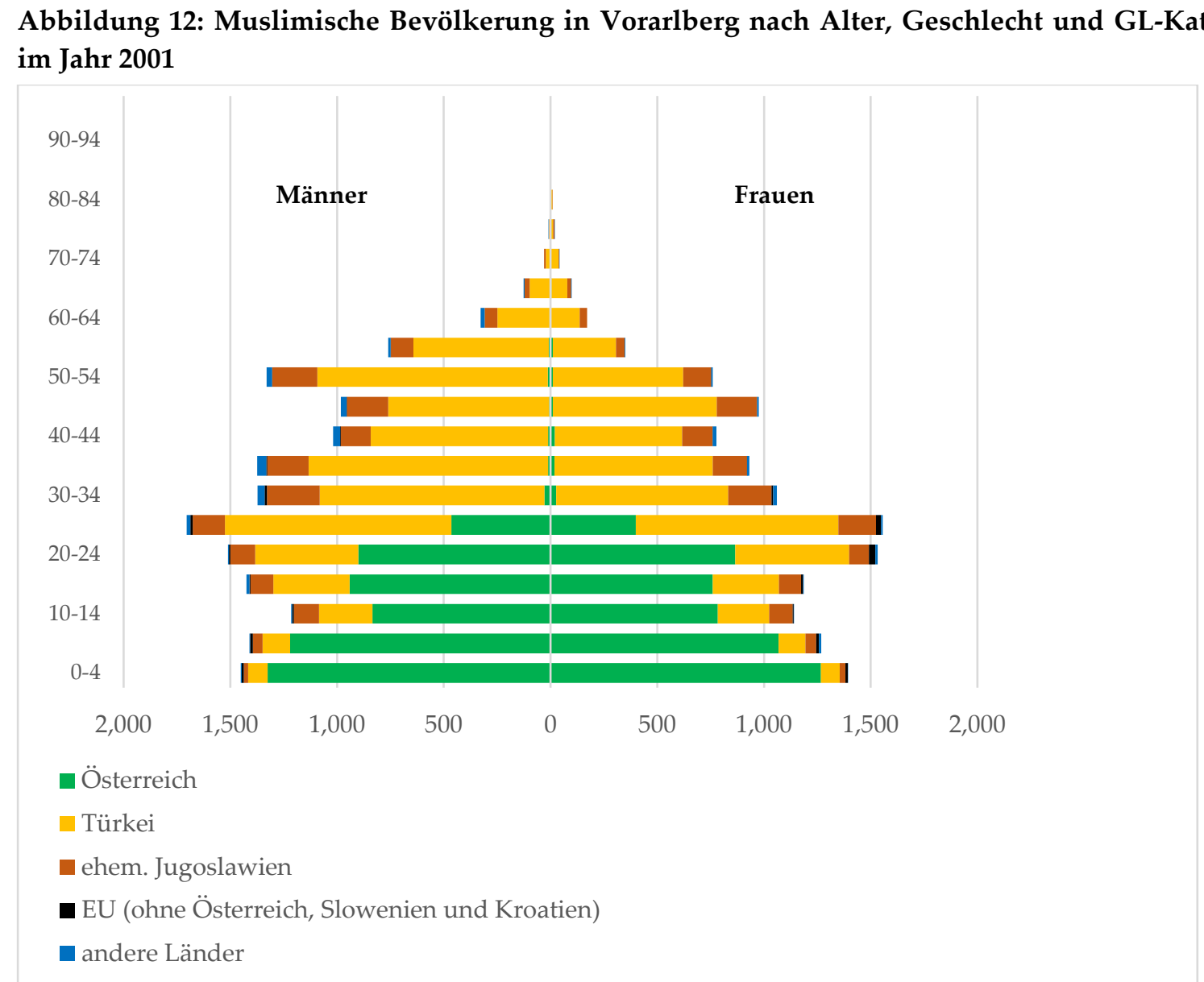

Quelle: Statistik Austria 


\subsection{Muslimische $\mathrm{Zu}$ - und Abwanderung in Vorarlberg}

Unter Anwendung der random migrant assumption lässt sich feststellen, dass die Nettomigration (Zuwanderung minus Abwanderung) von Musliminnen und Muslimen nach Vorarlberg zwischen 2001 und 20179.527 Personen betrug. Die muslimische Zu- und Abwanderung in Vorarlberg folgt einem sehr ähnlichen Zeitmuster wie die Zu- und Abwanderung in Vorarlberg im Allgemeinen, jedoch mit stärkeren Auf- und Abschwüngen: Nach einem moderaten Rückgang zwischen 2003 und 2010 stieg die muslimische Nettomigration wieder an, nahm rasch zu und erreichte ihren Höhepunkt im Jahr 2015, wonach sie bis heute wieder stark abnahm.

Abbildung 13: Muslimische Migration nach und aus Vorarlberg, 2002-2017

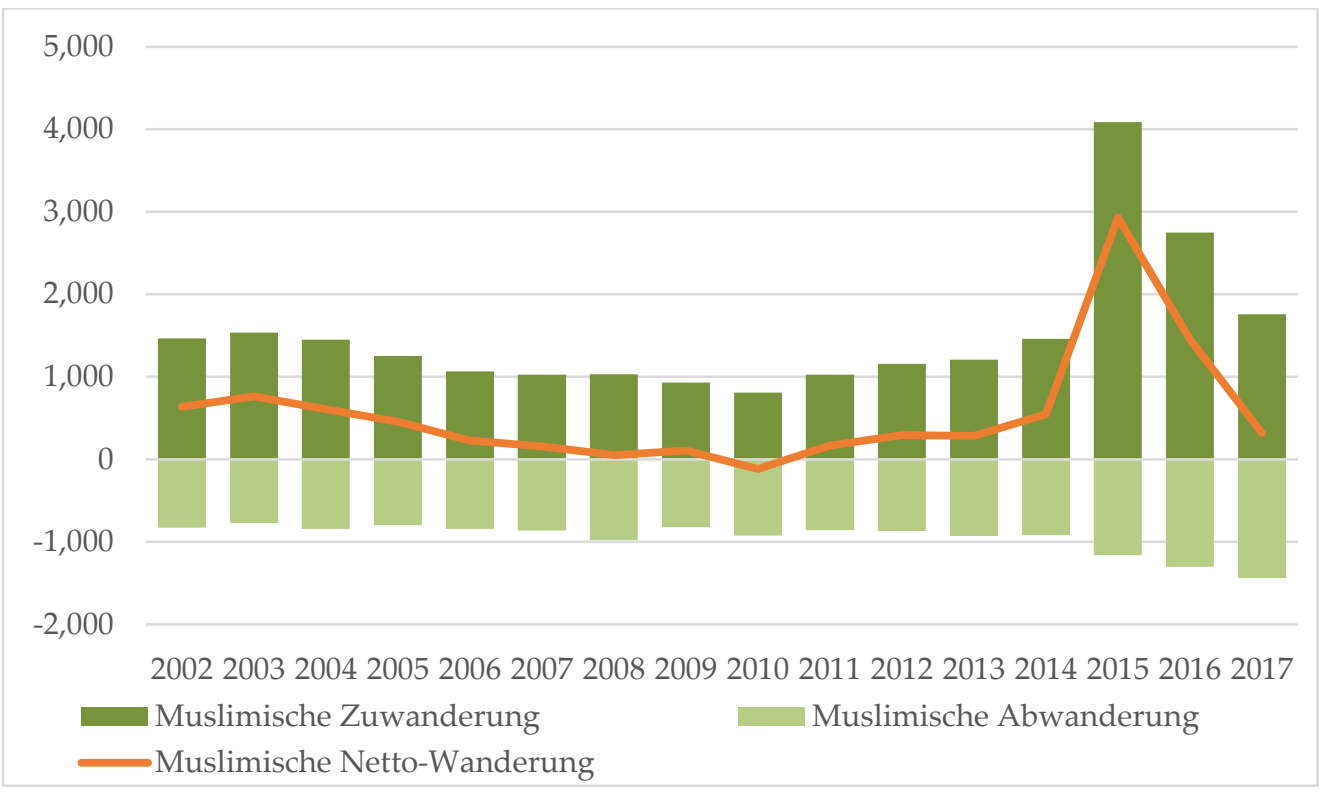

Quelle: Berechnung der Autorinnen auf der Grundlage von Daten der Statistik Austria

Betrachtet man nun die Herkunftsländer muslimischer Zuwanderinnen und Zuwanderer, so geht aus Abbildung 14 deutlich hervor, dass bei der Nettomigration nach Geburtsländern zwischen 2002 und 2017 starke Unterschiede in Volumen zu verzeichnen sind. Während bis 2007 der größte Teil der muslimischen Nettozuwanderinnen und -zuwanderer in der Türkei geboren wurden, findet danach eine deutliche Veränderung bei den Herkunftsländern statt. Die muslimische Zuwanderung aus außereuropäischen Ländern erfolgte weitgehend zwischen 2011 und 2017, insbesondere aus Ländern wie Syrien, Afghanistan und dem Irak. Dieses sich wandelnde Muster muslimischer Zuwanderung verändert natürlich auch die Zusammensetzung der muslimischen Bevölkerung in Vorarlberg im Jahr 2018 im Vergleich zum Jahr 2001. 
Abbildung 14: Muslimische Nettomigration nach Vorarlberg nach GL-Kategorien, 2002-2017

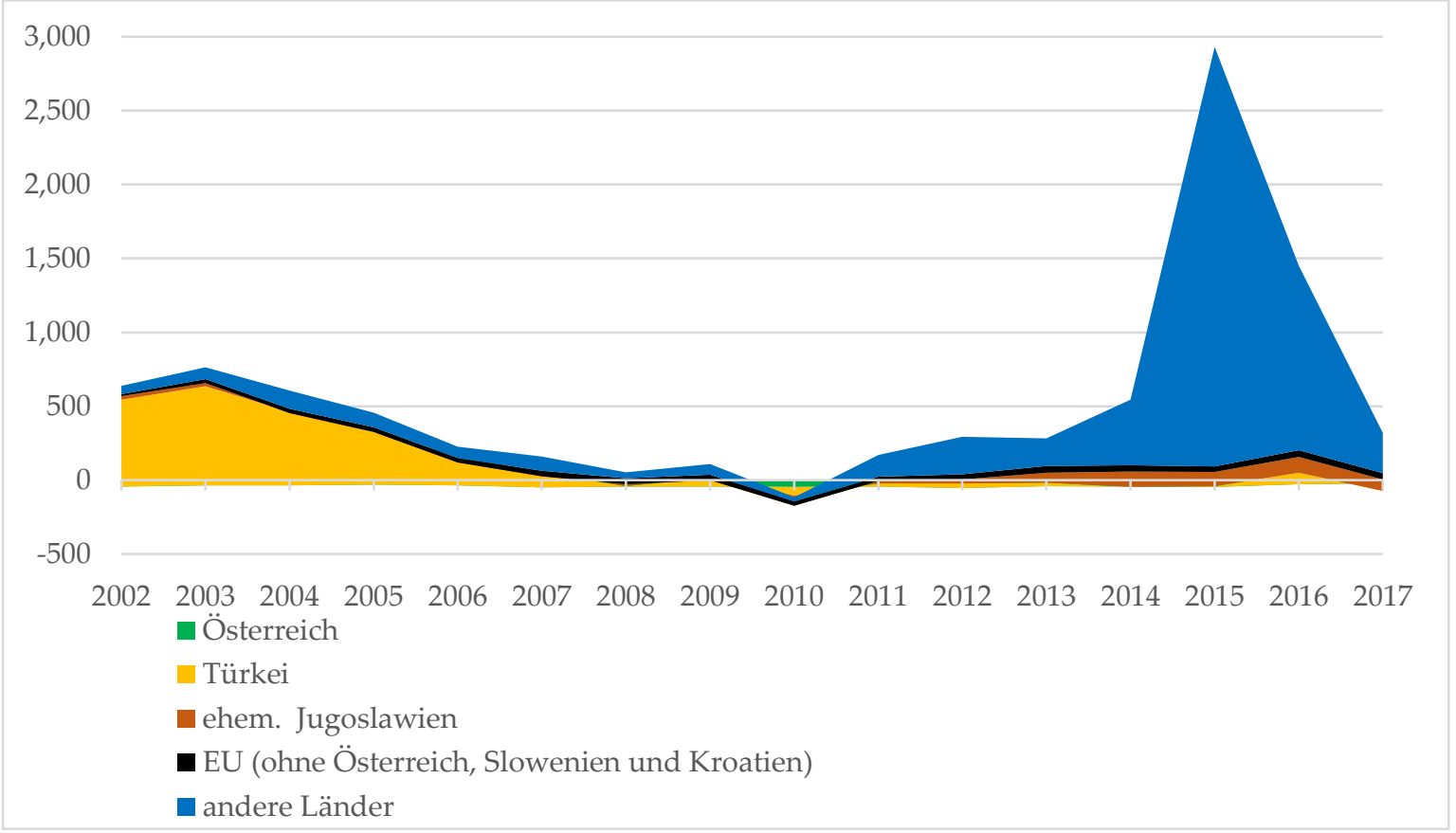

Quelle: Berechnung der Autorinnen auf der Grundlage von Daten der Statistik Austria

\subsection{Herkunftsländer der muslimischen Bevölkerung Vorarlbergs}

Seit 2001 sind erhebliche Veränderungen bei den Geburtsländern der Vorarlberger Musliminnen und Muslime $\mathrm{zu}$ verzeichnen. Weniger muslimische Glaubensangehörige wurden in der Türkei geboren (von 50 \% im Jahr 2001 auf 34 \% im Jahr 2018), mehr in Österreich (von $38 \%$ auf $45 \%$ ), und ein größerer Anteil der muslimischen Bevölkerung wurde in anderen Ländern als den traditionellen Ländern der muslimischen Migration nach Vorarlberg geboren, und zwar in Syrien und Afghanistan. 
Abbildung 15: Geburtsländer der muslimischen Bevölkerung Vorarlbergs nach Länderkategorien, 2001-2018
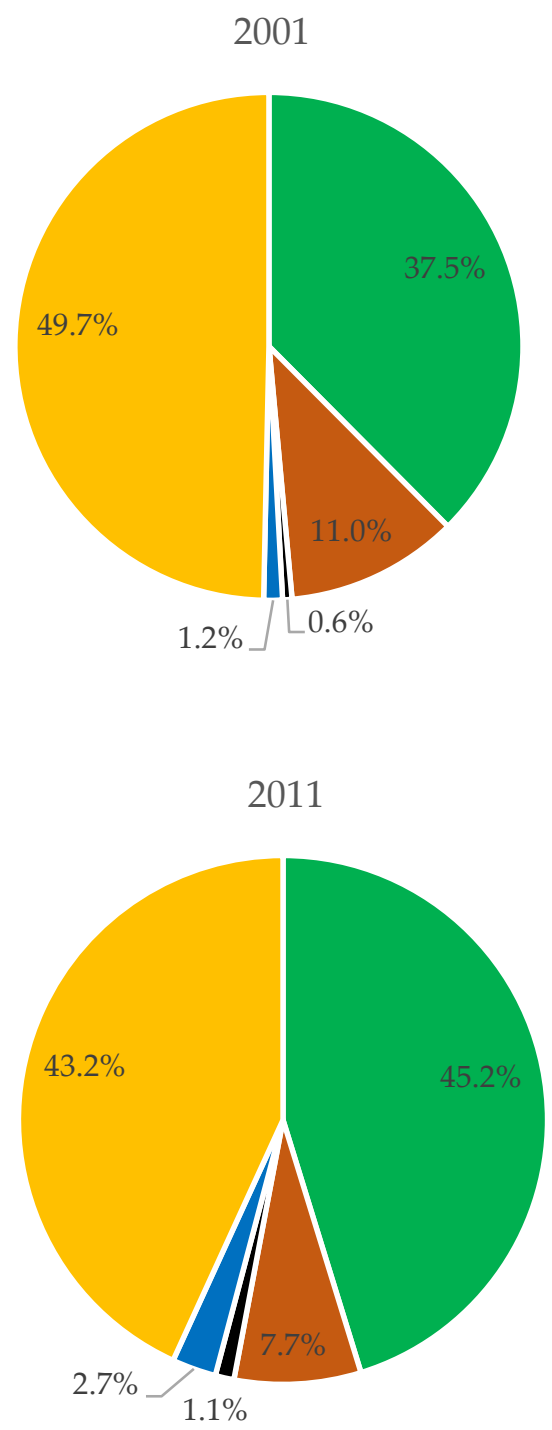

2018



2006

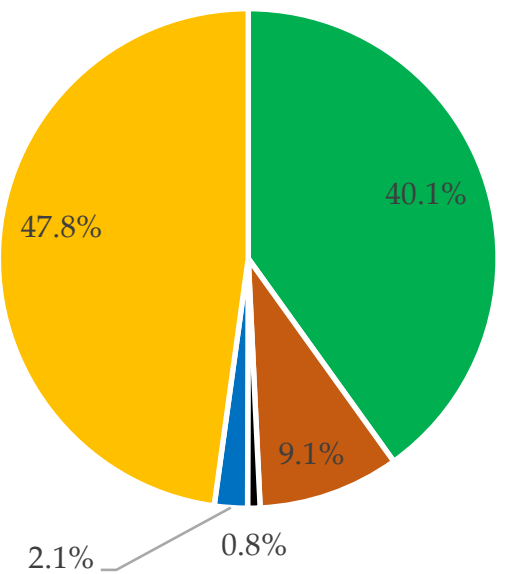

2016

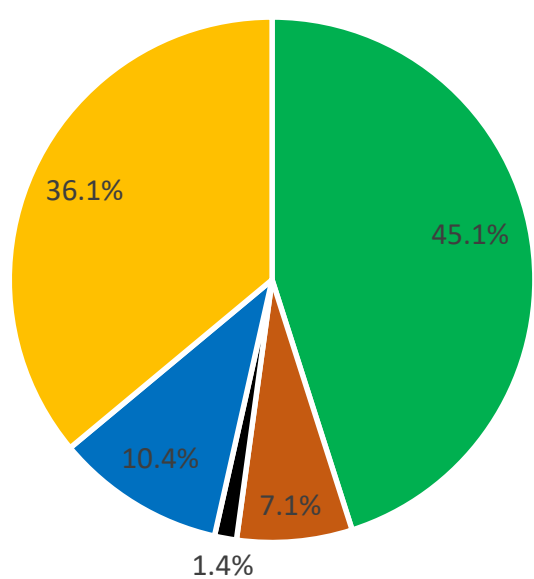

Österreich

Türkei

Ehem. Jugoslawien

EU (ohne Österreich, Slowenien und Kroatien)

andere Länder

Quelle: Berechnung der Autorinnen auf der Grundlage von Daten der Statistik Austria 
Abbildung 16 zeigt die Alterspyramide der im Jahr 2018 in Vorarlberg lebenden Musliminnen und Muslime nach Geburtsland. Vergleicht man sie mit der Alterszusammensetzung im Jahr 2001 (siehe Abbildung 12), zeigt sich eine Verstärkung des bereits beobachteten Musters, wobei große Teile der jüngeren Altersgruppen der zweiten Generation angehören. Es zeigt sich auch die Zuwanderung neuer und junger Musliminnen und Muslime in den letzten Jahren.

Abbildung 16: Muslimische Bevölkerung in Vorarlberg nach Alter, Geschlecht und GL-Kategorie im Jahr 2018

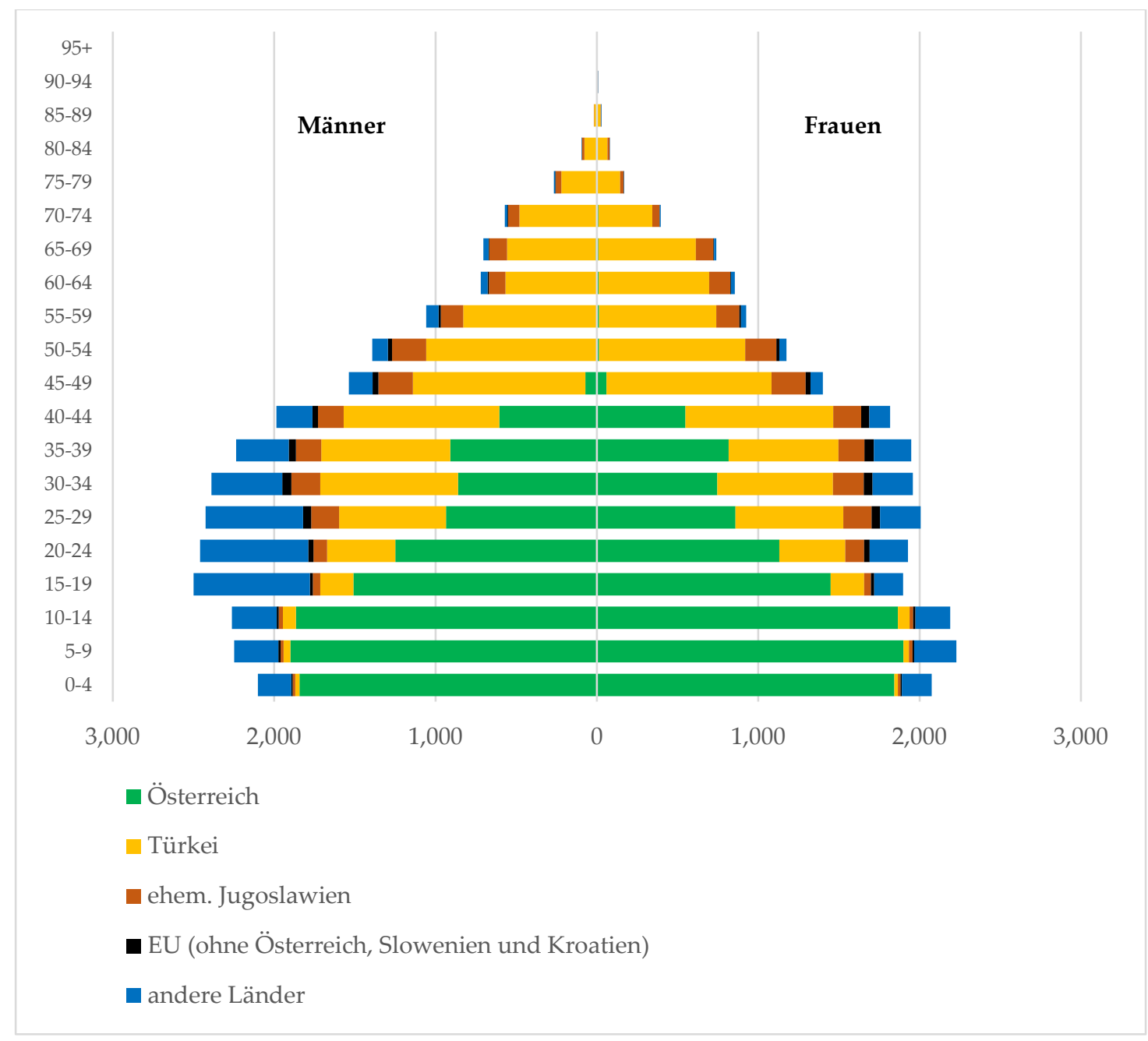

Quelle: Berechnung der Autorinnen auf der Grundlage von Daten der Statistik Austria 


\section{Fazit}

Die religiöse Landschaft Vorarlbergs hat sich nach unseren Schätzungen zwischen 2001 und 2018 zwar verändert, aber wahrscheinlich nicht so sehr, wie manche glauben möchten. Der Anteil der muslimischen Bevölkerung ist in den vergangenen 17 Jahren von 8,5\% auf $13 \%$ gestiegen. Die Veränderung in der Verteilung der Bevölkerung nach Religionsbekenntnissen ist nicht so deutlich wie in Wien. Dennoch haben römisch-katholische Glaubensangehörige deutlich weniger Gewicht in der Bevölkerung als zu Beginn des Jahrhunderts und die Anzahl der Personen ohne Bekenntnis hat sich zwischen 2001 und 2018 mehr als verdreifacht. Auch die Zusammensetzung der muslimischen Bevölkerung hat sich im Beobachtungszeitraum stark verändert: Musliminnen und Muslime der zweiten oder dritten Generation mit türkischem Familienhintergrund machen einen immer größeren Anteil aus. Es ist eine steigende Diversifizierung der muslimischen Glaubensangehörigen zu beobachten - vor allem aufgrund der Fluchtmigration der Jahre 2015 und 2016. Wie sich dies zukünftig entwickeln wird, könnte mit Bevölkerungsprojektionen untersucht werden, die nicht Teil dieser Arbeit waren. Die neuesten verfügbaren Daten zeigen einen starken Rückgang der Nettomigration nach Vorarlberg, ein Trend, der - wenn er sich fortsetzt - zu einer langsameren religiösen Diversifizierung in Vorarlberg führen könnte. 


\section{Literatur}

Buber-Ennser, I., Goujon, A., Kohlenberger, J., Rengs, B. (2018): Multi-Layered Roles of Religion among Refugees Arriving in Austria around 2015. Religions 2018, 9(5), 154. Verfügbar unter: doi.org/10.3390/rel9050154.

Buber-Ennser I., J. Kohlenberger, B. Rengs, Z. Al Zalak, A. Goujon, E. Striessnig, M. Potančoková, R. Gisser, M. R. Testa, W. Lutz (2016): Human Capital, Values, and Attitudes of Persons Seeking Refuge in Austria in 2015. PLoS ONE 11 (9). Verfügbar unter: https://journals.plos.org/plosone/article?id=10.1371/journal.pone.0163481.

Goujon et al. (2014): WIREL project key findings: Religions in Vienna in the past, present and future. Wien: Vienna Institute of Demography.

Goujon, A., S. Jurasszovich, M. Potancoková (2017): Demographie und Religion in Österreich: Szenarien 2016 bis 2046. ÖIF-Forschungsbericht. Wien: Österreichischer Integrationsfonds.

Kulu, H. and A. González-Ferrer (2014): Family dynamics among immigrants and their descendants in Europe: Current research and opportunities. European Journal of Population, 30(4): 411-435.

McQuillan, K. (2004): When Does Religion Influence Fertility? Population and Development Review, 30(1), 25-56. Verfügbar unter: http://www.jstor.org/stable/3401497.

PEW Research Center (2011): Global Christianity. Verfügbar unter: http://www.pewforum.org/2011/12/19/global-christianity-exec/.

PEW Research Center (2012): The Global Religious Landscape. Verfügbar unter: http://www.pewforum.org/2012/12/18/global-religious-landscape-exec/.

Toulemon, L. (2004): Fertility among immigrant women: new data, a new approach. Population and Societies, N. 400: 1-4. Verfügbar unter: http://www.demogr.mpg.de/papers/working/wp-2014-001.pdf. 